\title{
El Burnout en el personal de Enfermería: ¿De qué manera no quemarse?
}

María Isabel Espinosa Zepeda, * Mtra. Rosa A. Zarate Grajales** y Lic. Victoria Fernández García. ***

*Pasante de la Licenciatura en Enfermería y Obstetricia. ENEO-UNAM. Trabajo realizado como opción de Titulación por ingreso a actividad de Investigación, **Jefa de la División de Estudios de Posgrado. Responsable del Proyecto PAPIIT IN304907-2 y *** Consultora de la Fundación Mexicana para la Salud. Corresponsable del Proyecto PAPIIT IN304907.

El presente trabajo es una revisión de la literatura sobre los factores de riesgo para la aparición del Síndrome de Burnout en el personal de Enfermería. Este síndrome caracterizado por "agotamiento mental", "estrés laboral", "estrés emocional" y "desgaste profesional" , aparece según los estudios revisados como resultado de múltiples factores personales y organizacionales entre los que destacan el estado civil, el género, la edad, la antigüedad laboral, el coeficiente Enfermera paciente y el ambiente físico y organizacional del trabajo. Así también se incluye algunas recomendaciones para que las organizaciones de salud y los profesionales de Enfermería puedan identificar oportunamente el Síndrome de Burnout, prevenir su aparición y definir acciones en consecuencia.

PALABRAS CLAVE: Síndrome de Burnout, Enfermería, Estrés.

\section{Alostract}

The present paper is a literature review about the risk factors for the appearance of burnout syndrome caused by "mental draining", "work stress", "emotional stress", and "professional draining"; according to the reviewed studies, it appears as a result of multiple personal and organizational factors, among which the most outstanding are the civil status, gender, age, time at work, the patient/nurse coefficient, and the physical and organizational environment at job site. Some recommendations are also included so health institutions and nursing professionals can timely identify the Burnout Syndrome", prevent its appearance and define actions accordingly.

KEY WORDS: Burnout Syndrome, Nursing, Stress.

\section{INTRODUCCIÓN}

El cuidar, es una actividad indispensable para la supervivencia. Desde que la humanidad existe, el cuidado ha sido relevante constituyendo una función primordial para promover y desarrollar todas aquellas actividades que hacen vivir a las personas y a los grupos. Sin embargo, a través del tiempo, el cuidar se ha convertido en la función exclusiva de un gremio, la profesión de Enfermería. Para Marie Francoise Collière "cuidar es mantener la vida asegurando la satisfacción de un conjunto de necesidades", "cuidar representa una serie de actos de vida que tienen por finalidad y por función mantener a los seres vivos para permitirles reproducirse y perpetuar la vida".'
En este sentido, la Enfermería brinda diferentes tipos de cuidados como los dirigidos a la curación, a limitar la enfermedad y combatir sus causas. En estas situaciones, el personal de Enfermería frecuentemente enfrenta demandas excesivas por parte de los pacientes, incluyendo las emocionales que en una situación crónica puede afectar a la salud. De esta manera, el estrés se convierte en una de las principales patologías del ámbito laboral-profesional para el personal de Enfermería. ${ }^{2}$

El estrés es la respuesta del cuerpo a condiciones externas que perturban el equilibrio emocional de la persona ${ }^{3}$. Una 
situación de estrés continuado debilita el sistema inmunológico debido a la acción de la cortisona que reduce la capacidad del cuerpo para combatir la infección y aumenta la susceptibilidad a determinadas enfermedades.

El síndrome de burnout (SB) o desgaste profesional se considera como una etapa avanzada del estrés laboral derivada de un estado crónico afectando la salud de los profesionales del área de la salud, la productividad en la organización así como en la salud de los pacientes. De esta manera, el profesional de Enfermería estaría limitado para brindar un cuidado de calidad.

\section{SÍNDROME DE BURNOUT}

El SB es un término inglés que se traduce como "quemadura" y cuyo uso en español ha sido "agotamiento mental", "estrés laboral", "estrés emocional", "desgaste profesional", entre otros ${ }^{4}$.

Freudenberger, describió por primera vez este síndrome, y lo definió como burnout o "quemarse" en referencia a la relación que se establece entre el individuo y su trabajo ${ }^{5}$. Al no cumplirse las expectativas de los profesionales se genera un "quemando" paulatino. Otra definición formulada por Pines, et al definen al SB como un "estado de agotamiento mental, físico y emocional producido por el involucramiento crónico en el trabajo en situaciones emocionalmente demandantes".6

Posteriormente Cristina Maslach, desarrolló este término describiendo respuestas emocionales de aquellos profesionales que trabajaban en sectores de ayuda como en el caso de la salud.

Este síndrome, según Maslach está integrado por tres componentes:

- Agotamiento emocional

- Despersonalización

- Falta de realización personal

El primero se define como la pérdida de energía tanto física como psicológica, manifestándose en agotamiento emocional (AE) para atender las demandas de las personas a las que se debe prestar asistencia.

El segundo aspecto es la despersonalización (DP), en la que se produce un cambio negativo en las actitudes hacia otras personas, conduciendo a los profesionales a pensar que los demás son, merecedores de los problemas que enfrentan.
El tercer componente es el sentimiento de falta de realización personal (RPD), apareciendo una serie de respuestas negativas hacia sí mismo y su trabajo, típicas de depresión: incremento de la irritabilidad, pérdida de la motivación hacia el trabajo, y baja autoestima, entre otras.

Mingote citado por Rogelia Perea, considera que es el burnout la consecuencia de un fracaso de adaptación individual por desgaste y agotamiento de los recursos psicológicos para el afrontamiento de las demandas del rol ocupacional directa con personas y que se caracteriza por cinco factores:

1. Conducta anormal del rol profesional con despersonalización, ausentismo y conflictos de relación con los compañeros de trabajo.

2. Síntomas disfóricos, sobre todo de malestar y agotamiento emocional.

3. Síntomas físicos de estrés, asociadas al afrontamiento disfuncional de las demandas del rol laboral.

4. Disminución del rendimiento laboral por desmotivación y pérdida de interés por el trabajo.

5. Inadecuada adaptación al trabajo producido por baja realización y pérdida de autoestima personal.

\section{FACTORES DE RIESGO}

El SB es una respuesta del individuo, cuando tiene que enfrentarse a diferentes situaciones que no se pueden controlar. Para que el síndrome se produzca es necesaria la interacción entre respuestas y características individuales al estrés, así como la presión laboral en el ambiente de trabajo; al respecto existen evidencias nacionales e internacionales sobre los siguientes factores de riesgo.

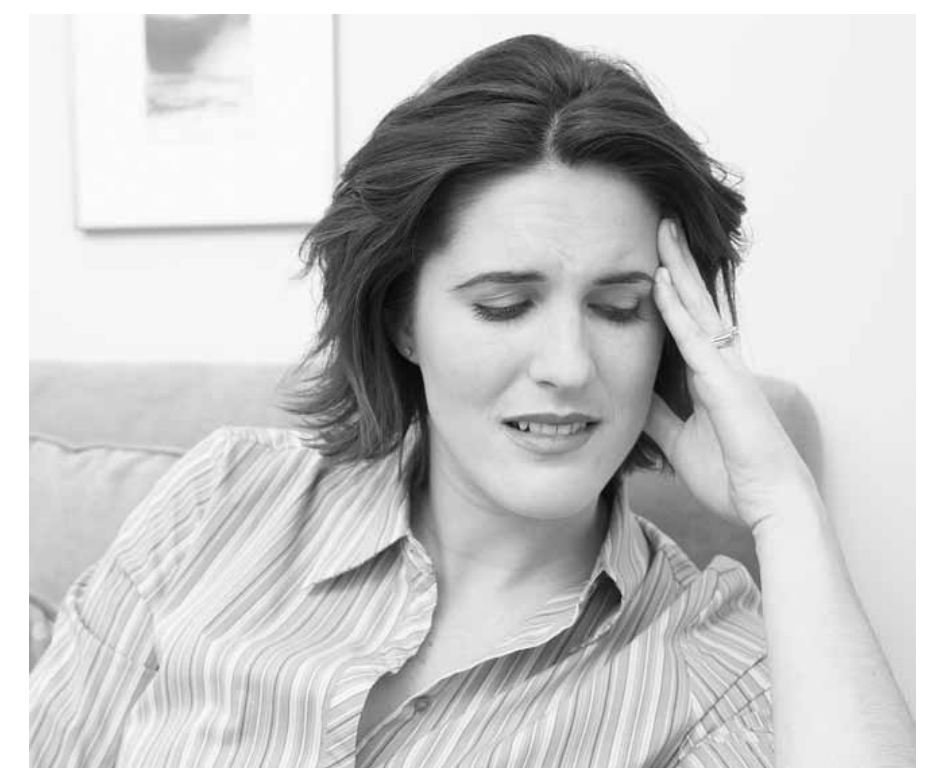


- $\quad$ Personalidad: Freudenberger consideraba teóricamente a las personas con alto riesgo de "quemar-

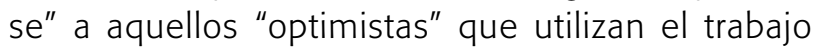
para tener una "buena opinión de si mismos", 7 por el contrario, Solano encontró que el ser optimista amortigua los síntomas de estar quemado ${ }^{8}$.

- $\quad$ En otro estudio Cristina Ortega refiere que los profesionales con inseguridad y problemas de autoestima tienen menor capacidad para afrontar la tensión y mayor riesgo de adaptación y de enfermar ${ }^{9}$.

- $\quad$ Edad: J. Carlos Atance y Martínez observaron que conforme la edad aumenta se produce mayor tensión emocional por la falta de actualización de conocimientos ${ }^{10},{ }^{11}$, así como Romana Albadejo, Rodolfo Quiroz y Carmen Perezagua en sus investigaciones refieren que la edad no tiene relación significativa ${ }^{12},{ }^{13},{ }^{14}$.

- $\quad$ Antigüedad: Al respecto María Solano, J. Carlos Atance y M. A. Caballero hacen referencia que a mayor antigüedad laboral, se genera mayor despersonalización y agotamiento emocional lo cual provoca el $\mathrm{SB}^{15}$ y refieren que los años promedio para que se de esta situación son de más de 15 a 19 años laborando, sin embargo Carmen Perezagua, E. A Adali y Pedro Gil-Monte han encontrado que las personas en sus primeros años laborales, al no contar con la experiencia necesaria genera estrés ante una situación desconocida durante el proceso de adaptación ${ }^{16},{ }^{17}$ haciéndolas más vulnerables al equilibrar las expectativas idealistas ante un contexto nuevo.

- Género: María Solano, C. Martínez López y Pedro Gil-Monte consideran como un grupo vulnerable a las mujeres debido a la doble carga de trabajo que conlleva la práctica profesional y la tarea familiar, sin embargo Armand Grau y Jorge Román encontraron que el género más vulnerable es el hombre ${ }^{18},{ }^{19}$ y refieren que en los hospitales en su mayoría el personal es femenino y no existe equidad para proporcionar datos con suficiente validez.

- Estado civil: Al respecto J. Carlos Atance, Carmen Perezagua y M. A. Caballero encontraron que el estar casado de alguna manera conlleva a una vida familiar rica actuando como amortiguador emocional.

- Tener hijos: Carmen Perezagua y M. A. Caballero encontraron que al tener hijos las personas son menos propensas a padecer el síndrome de burnout, interpretando los mismos autores que esta condición las hace más realistas y maduras y con mayores posibilidades de afrontar conflictos emocionales; sin embargo Armand Grau, Romana Albadejo y Pedro Gil-Monte mencionan que no se encontró relación significativa en esta área con dicho padecimiento ${ }^{20}$

- Horas de trabajo: J. Carlos Atance y Rodolfo Quiroz encontraron mayor cansancio emocional en aquellos profesionales que dedican entre 36 y 40 horas a la semana, con respecto a los que trabajan menos de 36 horas sin embargo María Solano y Pedro Gil-Monte no encontraron relación entre este factor y el SB.

- Horario de trabajo: Las referencias revisadas de María Solano y Pedro Gil-Monte muestran que el SB se da más en aquellas personas que trabajan en el turno nocturno, seguido por el vespertino y por último el matutino esto se ha explicado por el hecho de que no hay una relación activa con el paciente encontrándose dormido la mayoría del turno y no existe reconocimiento por parte de él, hacia las intervenciones que la enfermera le brinda; sin embargo M. A. Caballero y Rodolfo Quiroz no encontraron relación con esta categoría y el SB.

- Situación laboral: M. A. Caballero muestra que hay mayor cansancio emocional en aquellos profesionales con plazas de base obteniendo puntuaciones más altas debido a la mayor carga laboral, por otro lado N. Ibáñez Y Jorge Román han observado que los profesionales suplentes o de contrato tienden más a la despersonalización. Esto se ha considerado así ya que no se ven realizadas las altas expectativas generadas al ingresar al centro de trabajo.

- Número de pacientes: J. Carlos Atance y María Solano refieren puntuaciones mayores en la dimensión de cansancio emocional en aquellos que tenían más de 20 pacientes haciendo referencia que existe la insatisfacción de no realizar las actividades en su totalidad no obteniendo los resultados que ellos esperaban en la atención al paciente.

- Nivel educativo: María Solano y E. A. Adali encontraron que aquellos que tienen un nivel académico más alto presentan niveles de moderado - alto ya que tienen mayor responsabilidad en cuanto a conocimientos.

Reconocimiento profesional: Armand Grau y Romana Albadejo refieren que existe una relación significativa 
para adquirir el SB, teniendo en cuenta que el personal de enfermería no percibe una expresión de recompensa verbal por parte de los pacientes así como de las autoridades ya sea de manera material o verbal, refiriendo los profesionales que su trabajo no es proporcional a lo que ellos dan, causando despersonalización.

- Áreas laborales: María Solano y Jorge Román hacen referencia que se presenta mayor cansancio emocional en los profesionales ubicados en áreas donde se requiere dar cuidados específicos requiriendo mayor numero de horas de atención al paciente como son $\mathrm{UCl}$, Oncología y Urgencias.

- $\quad$ Tiempo libre: Rodolfo Quiroz y N. Ibañez encontraron que el personal de Enfermería que cuenta con menos de 6 horas semanales de ocio o tiempo libre para otras actividades son mas propensas a la despersonalización $y$ al agotamiento emocional explicando que tienen menos tiempo para liberase de la tensiones del trabajo.

\section{MANIFESTACIONES}

Es importante resaltar que el SB no se presenta de manera súbita, este emerge de forma paulatina, con un incremento progresivo en la severidad. Para su estudio se han identificado tres fases:

- $\quad$ Primera: donde las demandas laborales exceden los recursos materiales y humanos, conduciendo a situaciones de estrés.

- Segunda: se presenta exceso de trabajo; donde el sujeto reacciona con una respuesta inadecuada a ese desajuste, apareciendo signos de ansiedad, fatiga, irritabilidad, significando un primer esfuerzo defensivo para adaptarse y sobrevivir ante lo que percibe como una situación crítica en la que hay una perdida de control.

- Tercera: donde se realiza un afrontamiento defensivo, aparecen cambios de actitudes y conductas con fin de defenderse activamente y contrarrestar las tensiones experimentadas. Las más significativas son: distanciamiento emocional, deseo de huida, cinismo y rigidez, entre otras.

\section{MEDICIÓN DEL SB}

El instrumento utilizado con mayor frecuencia para medir $\mathrm{SB}$, es el "Maslach Burnout Inventory" (MBI) (Maslach y
Jackson, 1981/1986); en su versión para los profesionales cuyo objeto de trabajo son personas) MBI-HSS es la versión clásica del $\mathrm{MBI}$ siendo más utilizado para medir el síndrome de Burnout, y se compone de 22 ítems que se distribuyen en tres escalas y miden la frecuencia con los que los profesionales perciben baja realización personal en el trabajo (8 ítems), agotamiento emocional (9 ítems), y despersonalización (5 ítems).

Para diagnosticar al profesional con $\mathrm{SB}$, el resultado del instrumento tendrá un valor alto en el componente de Cansancio Emocional (CE) y Despersonalización (DP), mientras que un valor bajo en Realización Personal (RP). Los profesionales por encima del percentil 75 se clasificarán en la categoría "alto", entre el percentil 75 y 25 en la categoría de "medio" y por debajo del percentil 25 en la categoría "bajo" de la puntuación total.

\section{FORMAS DE AFRONTAMIENTO A NIVEL INDIVIDUAL DEL SB}

Cuando los profesionales de Enfermería se ven afectados por este síndrome empiezan a sentirse irritables, aumentan las ausencias laborales, baja la calidad de atención y presentan trastornos del sueño. También es frecuente la dificultad para concentrarse debido a la ansiedad experimentada, produciéndose así un descenso del rendimiento laboral, al mismo tiempo surgen dudas acerca de su propia competencia profesional, con el consiguiente descenso en su autoestima, y otros síntomas llevándolos a toxicomanías ${ }^{20}$.

Los síntomas defensivos aluden a la negación emocional, tratándose de un mecanismo que utiliza el profesional para poder aceptar sus sentimientos, negando las emociones anteriormente descritas cuyas formas más habituales son: negación de las emociones, ironía, atención selectiva y el desplazamiento de sentimientos hacia otras situaciones o cosas. También pueden utilizar para defenderse la intelectualización o la atención parcial hacia lo que le resulta menos desagradable ${ }^{21}$.

\section{DISCUSIÓN Y CONCLUSIONES}

De primera instancia cabe señalar que se necesita realizar investigación en la población mexicana para crear y plantear intervenciones y que generen modelos para delimitar este síndrome teniendo suficiente evidencia.

Por otro lado conocer los problemas que acompañan el desarrollo de los profesionales de Enfermería, posibilita 
el desarrollo de estrategias específicas de afrontamiento que devuelvan el equilibrio y nos permitan mantener la satisfacción en el cumplimiento de las tareas de la profesión, así como considerar de gran importancia la "salud" de los profesionales de Enfermería que proporcionan el cuidado a personas, y disminuir los costos económicos y sociales que supone tener personal "quemado", ahora es cuestión de intervenir.

La prevención del estrés profesional puede iniciarse en las mismas unidades de trabajo. Las acciones preventivas y las estrategias de intervención se deben realizar, teniendo un plan de acción que abarque tres dimensiones: la personal, la grupal y la organizacional que considere todos los factores de riesgo antes mencionados.

\section{ASPECTOS PERSONALES}

- La adquisición de habilidades para la resolución de problemas preparan mejor al personal para identificar factores de estrés que puedan conducir al Burnout, como: asertividad, manejo del tiempo, entre otros.

- La Terapia Floral es un recurso que se centra armonizando las experiencias emocionales negativas, aumenta la sensibilidad del profesional hacia los pacientes y contribuye al ajuste de la autopercepción del nivel de realización profesional alcanzado 22 .

- Conseguir un equilibrio personal con técnicas que refuercen el optimismo y la autoestima traduciéndose en una mejor relación con los demás.

\section{ASPECTOS A NIVEL GRUPAL}

- Utilizar los canales de comunicación que haya en la empresa: para informar a los superiores de las preocupaciones que los inquietan.

- Delimitar las funciones: para evitar la sobrecarga de trabajo.

\section{ASPECTOS ORGANIZACIONALES}

- La organización deberá tener en cuenta la importancia del entorno físico como elemento estresante, proporcionando al trabajador espacios que le permitan tiempo de descanso.

- $\quad$ Favorecer que los profesionales tengan oportunidad para acceder a la educación continua ya que estimula y prepara al profesional en su práctica cotidiana con sistemas de evaluación y retroalimentación.

- Participación responsable en la elaboración de planes de trabajo como el aumento de equidad en la distri- bución del trabajo y contar con recursos materiales suficientes.

- $\quad$ 'Favorecer espacios donde se proporcione asesoramiento y ayuda psicológica a equipos de trabajo o personal de "alto riesgo" (oncología, unidades de cuidados intensivos, unidades de quemados, etc.).

\section{NOTA}

Cabe mencionar las diferentes versiones comercializadas del MBI: a) MBI- Human Services Survey (MBI-HSS), dirigido a los profesionales de la salud, b) $\mathrm{MBI}$ - Educators Survey (MBI-ES) para profesionales de la educación, y c) el MBI- General Survey (MBI-GS), presenta un carácter más genérico no exclusivo para profesionales cuyo objeto de trabajo son personas.

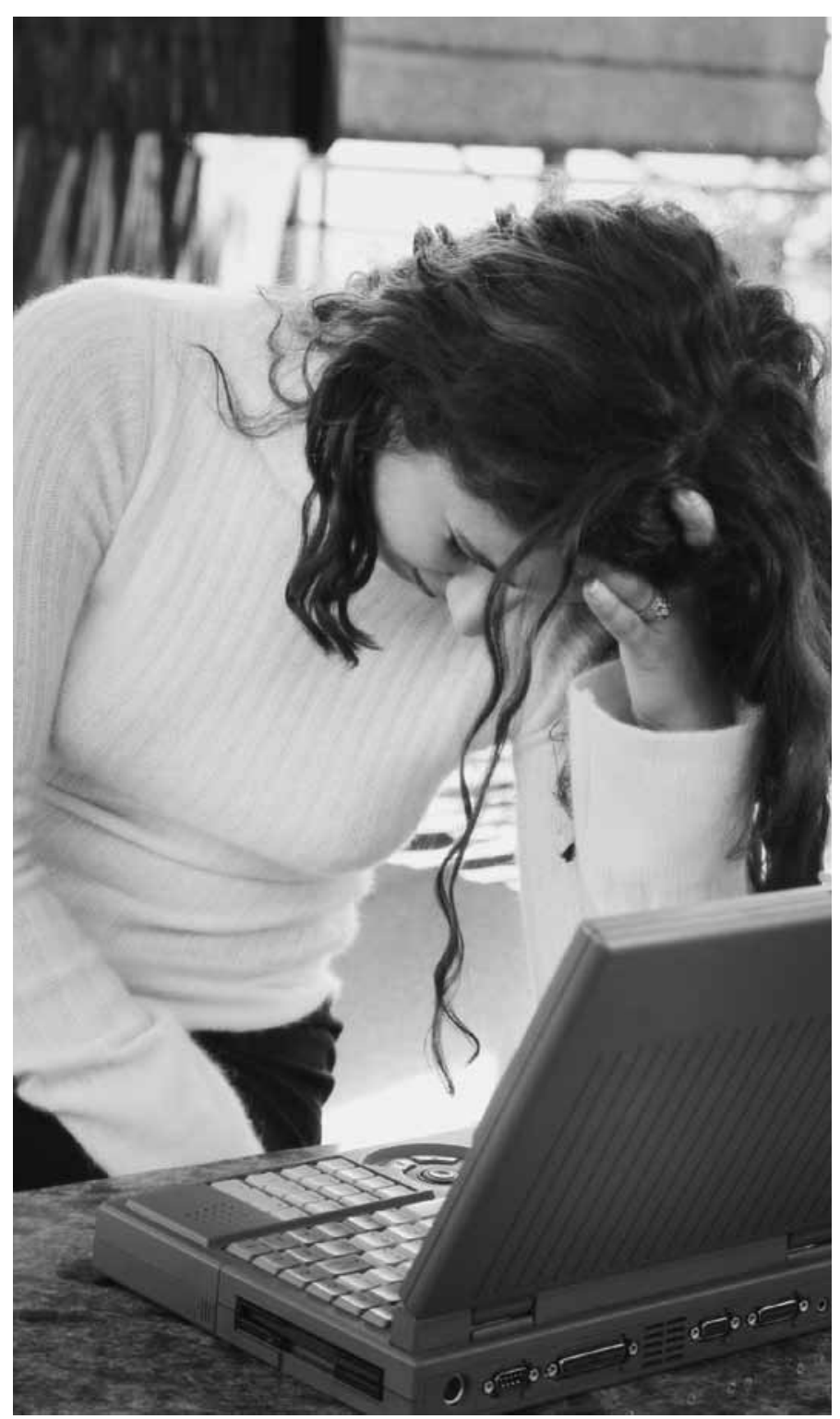




\section{REFERENCIAS BIBLIOGRÁFICAS}

1 Collière, MF. Promover la vida; de la práctica de las mujeres cuidadoras a los cuidados de enfermería. México. Ed. Mc Graw-Hill Interamericana. 1997. p. 285-337.

2 Medina - Chamorro; F. Salud Laboral. En: Frías - Osuna; A. Salud pública y educación para la salud. Madrid: Ed. Masson; 2000. p. 327-337.

3 Lluch, M. Novel, G. y Ortiz, A. La Ansiedad y el Estrés como componentes básicos del enfermar. En: Novel, G. Lluch, M, y López, D. Enfermería Psicosocial y Salud Mental. España: Ed. Masson; 2005 p. $165-173$.

4 Perea - Quesada; R. La salud en el ámbito laboral. En: Perea Quesada R. Educación para la salud; reto de nuestro tiempo. Madrid: Ed. Díaz de Santos; 2004. p. 181 - 197

5 Grau Armand y cols. Desgaste Profesional en el personal sanitario. Gaceta Sanitaria. 2005; 19 (6): p. 463-470.

6 Pines citado por Lluch, M. Novel, G. y Ortiz, A. op cit. 168.

7 Grau Armand y cols. op cit. p. 464.

8 Solano - Ruiz; MC, y cols. Síndrome de burnout en profesionales de enfermería de cuidados críticos. Enfermería Intensiva. 2002; 13 (1): p. 9-16.

9 Ortega - Ruiz; C, López - Ríos; F. El Burnout o síndrome de estar quemado en los profesionales sanitarios: revisión y perspectivas. International Journal of Clinical and Health Psychology. 2004; 4 (1): p.137-160.

10 Martínez - López; C, López - Solache; G. Características del síndrome de Burnout en un grupo de enfermeras mexicanas. Archivos de Medicina Familiar. 2005; 7 (1): p. 6-9.

11 Atance - Martínez; J. Aspectos epidemiológicos del síndrome de Burnout en el personal sanitario. Revista Española. Salud Pública. 1997; 71 (3): p. 293-303.

12 Albadejo, Romana, y cols. Síndrome de burnout en el personal de enfermería de un hospital de Madrid. Revista Española. Salud Pública. 2004; 78 (4): p.505-516.

13 Quiroz - Valdivia; R, Saco - Méndez; S. Factores Asociados al Síndrome de Burnout en Médicos y enfermeras del Hospital Nacional Sur Este de ESSALUD del CusCo. SITUA. 2003; 12 (23): p. 11-22

14 Perezagua - García; MC, Del Río - Moro; O, Vidal - Gómez; B. Burnout en enfermeras del Hospital Virgen de la Salud: Enfermeras de Urgencias vs. Enfermeras resto hospital. Enfermería en Cardiología. 2003; 29: p. 24 - 29.
15 Caballero - Martín; M, y cols. Prevalencia y factores asociados al burnout en un área de salud. Atención Primaria. 2001; 27 (1): p. 313-317.

16 Adali EA, y cols. Síndrome del Quemado en el personal de enfermería psiquiátrica de hospitales griegos. Eur. J. Psychiat. (Ed. Esp.). 2003; 17 (3): p. 161-170.

17 Gil - Monte PR. El síndrome de Quemarse por el trabajo (Síndrome de Burnout) en profesionales de enfermería. Revista Electrónica InterAcao. Psy. 2003; 1 (1): p. 19-33

18 Ibáñez - Martínez; N, Vilaregut - Puigdesens; A, Abio - Roig; A. Prevalencia del síndrome de burnout y el estado general de salud en un grupo de enfermeras de un hospital de Barcelona. Enfermería Clínica. 2004; 14 (3): p.142 - 151

19 Román - Hernández; J. Estrés y Burnout en profesionales de la salud de los niveles primario y secundario de atención. Revista Cubana Salud Pública. 2003; 29 (2): p. 103-110.

20 Gil - Monte PR. Validez Factorial de la adaptación al español del Maslach Burnout; Inventory - General Survey. Salud Pública de México. 2002; 44 (1): p. 33-40

21 Álvarez E, Fernández L. El Síndrome de «Burnout» o el desgaste profesional. I: Revisión de estudios. Revista de la Asociación Española de Neuropsiquiatría. 11(39):p. 991

22 Gil - Monte PR. Influencia de género sobre el proceso de desarrollo del síndrome de Quemarse por el trabajo (Burnout) en profesionales de Enfermería. Psicología em Estudo, Maringá. 2002; 7 (1): p. 3-10.

23 Pedroza - Jorge; E. (Enero 2004). Aproximación a la tecnología del estudio del burnout en mujeres dirigentes, [en línea]. Recuperado el 11 de noviembre 2006, de http://www.esenciasflorales.net/inicio/ARTICULO/burnout.htm.

\section{DIRECCIÓN PARA CORRESPONDENCIA:}

Ma. Isabel Espinosa Zepeda: eizileo@hotmail.com Mtra. Rosa A. Zarate Grajales: zarate_amarilis@hotmail.com Lic. Victoria Fernández García: vicky@funsalud.org.mx 